\title{
ZIC2 upregulates IncRNA SNHG12 expression to promote endometrial cancer cell proliferation and migration by activating the Notch signaling pathway
}

\author{
PENGYU CAI ${ }^{1 *}$, GAIJUAN LI ${ }^{2 *}$, MINGXIU WU ${ }^{1}$, BIN ZHANG $^{1}$ and HONG BAI ${ }^{3}$ \\ ${ }^{1}$ Department of Obstetrics and Gynecology, Dongguan People's Hospital, Dongguan, Guangdong 523000; \\ ${ }^{2}$ Department of Obstetrics and Gynecology, Midwifery Profession, Shanxi Health Vocational College, Jinzhong, \\ Shanxi 030600; ${ }^{3}$ The Second Ward of Gynecology, Dalian Obstetrics and Gynecology Hospital Affiliated to \\ Dalian Medical University and Dalian Maternal and Child Health Care Hospital, \\ Dalian, Liaoning 116033, P.R. China
}

Received January 15, 2021; Accepted May 25, 2021

DOI: $10.3892 / \mathrm{mmr} .2021 .12271$

\begin{abstract}
It was previously reported that long non-coding RNA (lncRNA) small nucleolar RNA host gene 12 (SNHG12) promoted the proliferation, invasion and migration of endometrial cancer (EC) cells; however, the upstream underlying mechanism remains unclear. The present study aimed to determine the possible underlying mechanism of SNHG12 regulating EC. The Encyclopedia of RNA Interactomes database was used to analyze whether SNHG12 could bind to Zic family member 2 (ZIC2) and the expression levels of ZIC2 in patients with EC.ZIC2 expression levels in EC cell lines were analyzed using western blotting and reverse transcription-quantitative PCR. RL95-2 cells were subsequently transfected with short hairpin RNA targeting ZIC2, or ZIC2 or SNHG12 overexpression plasmids. Cell proliferation, migration and invasion were analyzed using Cell Counting Kit-8, colony formation, wound healing and Transwell assays, respectively. The binding between ZIC2 and SHNG12 was verified using dual luciferase reporter and chromatin immunoprecipitation assays. The results of the present study revealed that the expression levels of ZIC2 were upregulated in the tissues of patients with EC and EC cell lines. ZIC2 knockdown inhibited RL95-2 cell proliferation, migration and invasion. The protein expression levels of Ki67, proliferating cell nuclear antigen, MMP2 and
\end{abstract}

Correspondence to: Dr Hong Bai, The Second Ward of Gynecology, Dalian Obstetrics and Gynecology Hospital Affiliated to Dalian Medical University and Dalian Maternal and Child Health Care Hospital, 1 Dunhuang Road, Dalian, Liaoning 116033, P.R. China

E-mail: baihongbh1@163.com

*Contributed equally

Key words: endometrial cancer, gynecology, long non-coding RNA small nucleolar RNA host gene 12, Notch signaling, Zic family member 2
MMP9 were also downregulated following the knockdown of ZIC2. ZIC2 was predicted to bind to SNHG12 and positively regulate SNHG12 expression. Further experiments demonstrated that the effects of the knockdown of ZIC2 on RL95-2 cells were partially reversed by SNHG12 overexpression. In addition, ZIC2 knockdown inhibited Notch signaling activation, while SNHG12 overexpression reversed this effect. In conclusion, the findings of the present study indicated that ZIC2 may upregulate SNHG12 expression to promote EC cell proliferation and migration by activating the Notch signaling pathway.

\section{Introduction}

Endometrial cancer (EC) comprises a group of epithelial malignant tumors that are found in the endometrium, which often occur in perimenopausal and postmenopausal women (1). EC is the 15th most common type of malignant tumor worldwide, and it is the most common gynecological malignant tumor in developed countries (2). Surgical resection and postoperative adjuvant treatments for EC have been standardized and comprise hysterectomy and bilateral salpingo-oophorectomy, followed by paclitaxel/platinum conventional chemotherapy (3). However, women with relapsed or advanced disease have a low response rate to conventional treatments and extremely poor clinical outcomes $(4,5)$. Therefore, it remains necessary to develop further targeted treatments for these patients based on the precise molecular pathogenesis of EC.

Our previous study demonstrated that long non-coding RNA (lncRNA) small nucleolar RNA host gene 12 (SNHG12) promoted cell proliferation, invasion and migration by targeting microRNA-4429 in EC (6). However, the upstream mediators and involved underlying mechanisms were not identified. Zic family member 2 (ZIC2) is a member of the ZIC family of $\mathrm{C} 2 \mathrm{H} 2$-type zinc finger proteins, which function as transcription factors and regulate tissue specific gene expression (7). A previous study reported that the differential expression of ZIC2 in EC was associated with lymph node 
metastasis (7). ZIC2 has also been indicated to serve oncogenic roles in numerous types of human cancer, including hepatocellular carcinoma (HCC) (8), cervical cancer (9), acute myeloid leukemia (10) and nasopharyngeal carcinoma (NPC) (11). However, to the best of our knowledge, the specific role of ZIC2 in EC has not been determined.

The IncRNA SNHG12 was demonstrated to promote cell proliferation and metastasis, and predicted a poor prognosis in NPC by modulating the Notch signaling pathway (12). In addition, the knockdown of SNHG12 inhibited the activation of epithelial-mesenchymal transition and Notch-1 signaling pathways in NPC (12). Dysregulated Notch signaling was discovered to affect the tumorigenicity and proliferation of cells in various types of cancer. For example, in EC, the expression levels of Notch1 and Notch3 were revealed to be upregulated and the elevated expression levels of Notch1 were associated with cancer aggressiveness and poor prognosis in patients (13). Blocking the Notch signaling pathway could also enhance the effect of the EGFR inhibitor by targeting CD133 ${ }^{+}$ EC cells (14). Therefore, the inhibition of the Notch signaling pathway may inhibit tumorigenesis and provide a novel therapeutic strategy for EC (14).

Notably, analysis using the JASPAR database predicted that ZIC2 could bind to the promoter of SNHG12. Therefore, the present study aimed to investigate whether ZIC2 could bind to SNHG12 to regulate EC cell proliferation and migration via activating Notch signaling.

\section{Materials and methods}

Cell lines and culture. The human EC cell lines, Ishikawa, KLE, RL95-2 and AN3 CA, were obtained from The Cell Bank of Type Culture Collection of the Chinese Academy of Sciences and the cultured human normal endometrial stromal cell (ESC) line (cat. no. BNCC340262; http://www.bnbio. com/pro/p1/1/p_3323.html) was obtained from BeNa Culture Collection; Beijing Beina Chunglian Institute of Biotechnology. All cells were cultured in DMEM (Sigma-Aldrich; Merck KGaA) supplemented with 15\% FBS (Sigma-Aldrich; Merck $\mathrm{KGaA}$ ) and $1 \%$ penicillin/streptomycin, and maintained in an atmosphere with $5 \% \mathrm{CO}_{2}$ at $37^{\circ} \mathrm{C}$.

Bioinformatics analysis. To analyze the overall level of ZIC2 differential expression between normal people and cancer patients, the clinical data in this study was obtained by searching the ENCORI database (15) from The Cancer Genome Atlas (TCGA) Genomics data (16), which is an authoritative online genome analysis technology dominantly based on large-scale sequencing, and at the same time, the information that can identify the patient in the clinical data is hidden.

Cell transfection. Short hairpin RNA (shRNA/sh) targeting ZIC2 (shRNA-ZIC2-1: 5'-CCGGCCGGAGTC TTTGAAGCTGAAACTCGAGTTTCAGCTTCAAAGACTCCGGTTTTTG-3'; and shRNA-ZIC2-2: 5'-CCGGCGGAA GCACATGAAGGTCC-ATCTCGAGATGGACCTTCATGT GCTTCCGTTTTTG-3') and scrambled negative control (NC) shRNA (shRNA-NC) (5'-ACTTGCGCTTGCGAAAATCTA TATAGC-3') were designed and synthesized by GenScript.
The ZIC2 and SNHG12 overexpression plasmids were established by inserting the full-length human ZIC2 and SNHG12 cDNA sequences (Aksomics, Inc.) into the pcDNA3.1 vector (Invitrogen; Thermo Fisher Scientific, Inc.); empty vectors served as the NCs (Oe-NC). All plasmids $(50 \mathrm{nM})$ were transfected for $2 \mathrm{~h}$ at $37^{\circ} \mathrm{C}$ into RL95-2 cells using Lipofectamine ${ }^{\circledR}$ 2000 reagent (Invitrogen; Thermo Fisher Scientific, Inc.) according to the manufacturer's protocol, and the transfection efficiency was evaluated at $48 \mathrm{~h}$ post-transfection using reverse transcription-quantitative PCR (RT-qPCR).

Cell counting Kit-8 (CCK-8) assay. RL95-2 cells were seeded at a density of $2 \times 10^{3}$ cells/well into 96 -well plates. Following transfection with the indicated vectors for 24,48 or $72 \mathrm{~h}$, the cells were incubated with $10 \mu \mathrm{l} \mathrm{CCK}-8$ solution (MedChemExpress) for $2 \mathrm{~h}$ at $37^{\circ} \mathrm{C}$. The absorbance of the cells was measured at a wavelength of $450 \mathrm{~nm}$ using a microplate reader.

Colony formation assay. For the colony formation assays, the RL95-2 cell suspension was resuspended in $1 \mathrm{ml}$ medium and then plated into 24 -well plates and incubated at $37^{\circ} \mathrm{C}$ for 2 weeks. Following incubation, cells were stained for $20 \mathrm{~min}$ at room temperature with $2 \%$ crystal violet and colonies (>50 cells) were counted under a light microscope (magnification, x100).

Wound healing assay. RL95-2 cells were seeded into a six-well plate at a density of $5 \times 10^{4}$ cells/well and cultured in an atmosphere with $5 \% \mathrm{CO}_{2}$ at $37^{\circ} \mathrm{C}$. Upon cell confluence reaching $70-80 \%$, the cell monolayer was scratched with a $100-\mu 1$ pipette tip to create an artificial wound. The cells were then cultured in serum-free DMEM for $24 \mathrm{~h}$ at $37^{\circ} \mathrm{C}$. Following incubation, cell migration was observed at 0 and $24 \mathrm{~h}$ using a light microscope (magnification, x100; Olympus Corporation). The cell migration rate $(\%)$ was calculated using the following equation: [(Initial distance at $0 \mathrm{~h}$-final distance at $24 \mathrm{~h}$ )/initial distance] $\mathrm{x} 100$.

Transwell assay. The Transwell assay was performed using 24-well culture plates with $8-\mu \mathrm{m}$ pore inserts (Falcon; Corning Life Sciences). The lower chamber was filled with $600 \mu 1$ DMEM supplemented with $10 \%$ FBS. The upper chamber was filled with $200 \mu$ l RL95-2 cell suspension ( $5 \times 10^{4}$ cells/well) suspended in serum-free DMEM. Following $24 \mathrm{~h}$ of incubation, the invasive cells were fixed with $4 \%$ methanol for $10 \mathrm{~min}$ at $37^{\circ} \mathrm{C}$ and then stained with $0.1 \%$ crystal violet solution for $15 \mathrm{~min}$ at $37^{\circ} \mathrm{C}$. The number of invasive cells in five randomly selected fields of view were counted using a counting chamber under a light microscope (magnification, x100).

Western blotting. Total protein was extracted from cells using RIPA lysis buffer (Beyotime Institute of Biotechnology). Total protein was quantified using a BCA kit (Thermo Fisher Scientific, Inc.) and $40 \mu \mathrm{g}$ protein/lane was separated via 8-12\% SDS-PAGE. The separated proteins were subsequently transferred onto PVDF membranes (Bio-Rad Laboratories, Inc.) and blocked with $5 \%$ non-fat milk at $37^{\circ} \mathrm{C}$ for $2 \mathrm{~h}$. The membranes were subsequently incubated with the following primary antibodies overnight at $4^{\circ} \mathrm{C}$ : Anti-ZIC2 (1:5,000; 
A
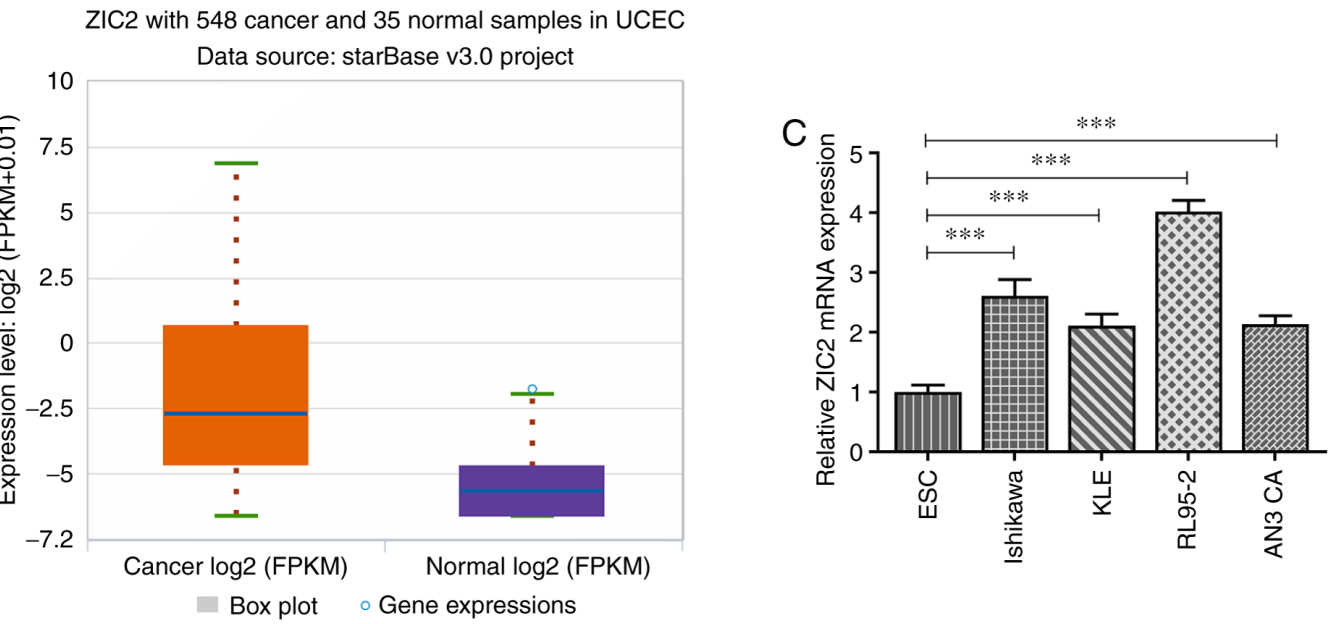

B
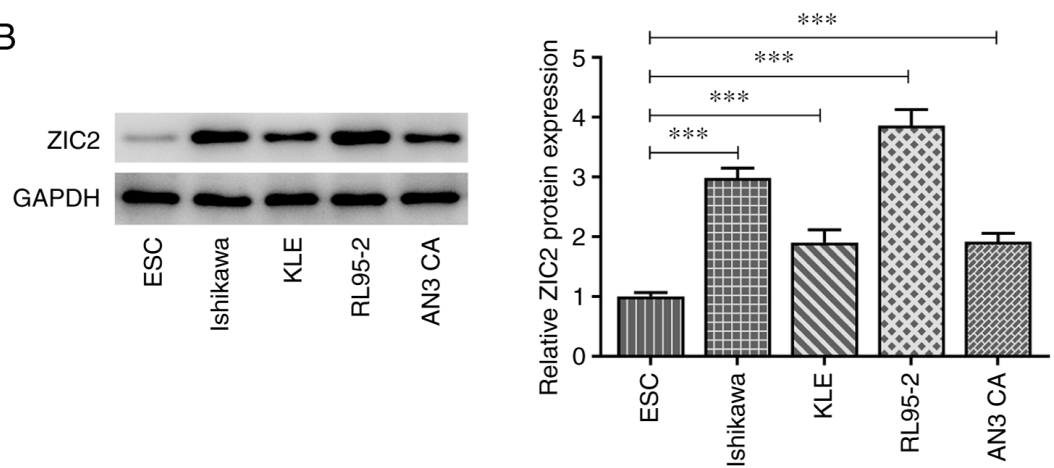

Figure 1. ZIC2 expression is upregulated in EC. (A) Expression levels of ZIC2 in patients with EC and normal tissues obtained from the ENCORI database. (B) Protein and (C) mRNA expression levels of ZIC2 in ESC cells and EC cell lines. ${ }^{* * *} \mathrm{P}<0.001$. ZIC2, Zic family member 2; EC, endometrial cancer; ENCORI, The Encyclopedia of RNA Interactomes; ESC, endometrial stromal cell.

cat. no. ab150404), anti-Ki67 (1:5,000; cat. no. ab92742), anti-proliferating cell nuclear antigen (PCNA; 1:1,000; cat. no. ab29), anti-MMP2 (1:1,000; cat. no. ab92536), anti-MMP9 (1:1,000; cat. no. ab137867), anti-activated Notch1 (1:500; cat. no. ab8925), anti-hes family bHLH transcription factor 1 (HES-1; 1:500; cat. no. ab108937) and anti-GAPDH (1:10,000; cat. no. ab181603; Abcam). Following the primary antibody incubation, the membranes were incubated with a goat anti-rabbit IgG HRP-conjugated secondary antibody (1:10,000; cat. no. ab205718; Abcam) at room temperature for $2 \mathrm{~h}$. Protein bands were visualized using an electrochemiluminescence kit (Invitrogen; Thermo Fisher Scientific, Inc.) and imaged on a gel imaging system (Bio-Rad Laboratories, Inc.). ImageJ software (version 1.52v; National Institutes of Health) was used for densitometric analysis.

$R T-q P C R$. Total RNA was extracted from cells using TRIzol ${ }^{\mathbb{B}}$ reagent (Invitrogen; Thermo Fisher Scientific, Inc.). Total RNA was reverse transcribed into cDNA using a PrimeScript cDNA Synthesis kit (Takara Biotechnology Co., Ltd.) according to the manufacturer's protocol. qPCR was subsequently performed using a SYBR Green Master mix (Takara Biotechnology Co., Ltd.) on an ABI 7500 Real-Time PCR system (Applied Biosystems; Thermo Fisher Scientific, Inc.). The following thermocycling conditions were used for the qPCR: Initial denaturation for $10 \mathrm{~min}$ at $95^{\circ} \mathrm{C}$; followed by 40 cycles of denaturation at $95^{\circ} \mathrm{C}$ for $20 \mathrm{sec}$, annealing at $60^{\circ} \mathrm{C}$ for $30 \mathrm{sec}$ and polymerization at $72^{\circ} \mathrm{C}$ for $20 \mathrm{sec}$. The following primer sequences were used for the qPCR: ZIC2 forward, 5'-AAG GACCCACACAGGGGAGAA-3' and reverse, 5'-AACATG ATCACAAGGTGCCCTC-3'; SNHG12 forward, 5'-TCTGGT GATCGAGGACTTCC-3' and reverse, 5'-ACCTCCTCAGTA TCACACACT-3'; and GAPDH forward, 5'-GAAAGCCTG CCGGTGACTAA-3' and reverse, 5'-TTCCCGTTCTCAGCC TTGAC-3'. The relative mRNA expression was calculated using the $2^{-\Delta \Delta C q}$ method (17). The expression levels of ZIC2 and SNHG12 were normalized to the expression levels of GAPDH.

Dual luciferase reporter assay. The JASPAR database (http://jaspar.genereg.net) was used to predict that ZIC2 could bind to the promoter of SNHG12. For the dual-luciferase reporter assay, wild-type (WT; 5'-GCAACAGCAGGATC-3') or mutant (MUT; 5'-ATGGTGATGAAGCT-3') SNHG12 sequences were amplified and cloned downstream of the luciferase reporter gene in pMIR-REPORT luciferase vectors (Thermo Fisher Scientific, Inc.). Cells were co-transfected with pcDNA3.1-ZIC2 or NC vectors and WT or MUT SNHG12 pMIR-REPORT luciferase vectors. Following $48 \mathrm{~h}$ of transfection, the relative luciferase activity was measured using a Dual Luciferase Reporter assay system (Promega Corporation). Luciferase activity was normalized to Renilla luciferase activity. All experiments were performed in triplicate.

Chromatin immunoprecipitation (ChIP) assay. ChIP assays were performed according to a standard protocol (18). Briefly, $5 \times 10^{7}$ RL95-2 cells were washed with cold PBS and then fixed 
A

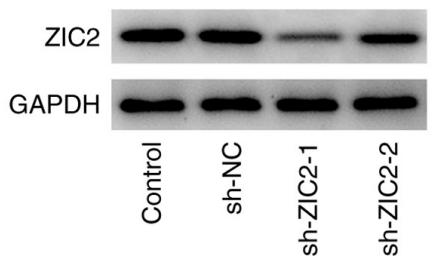

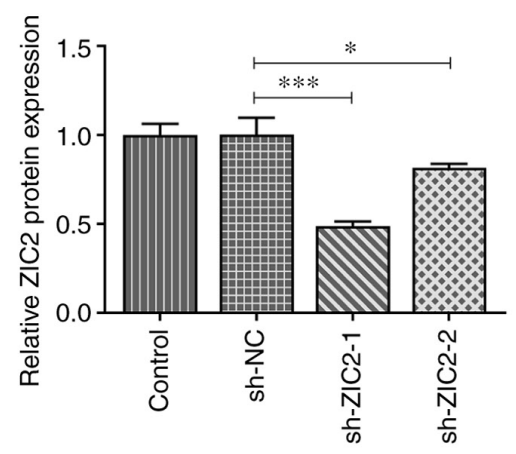

D
B

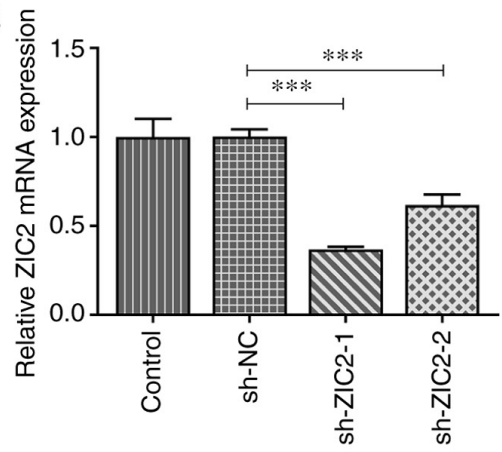

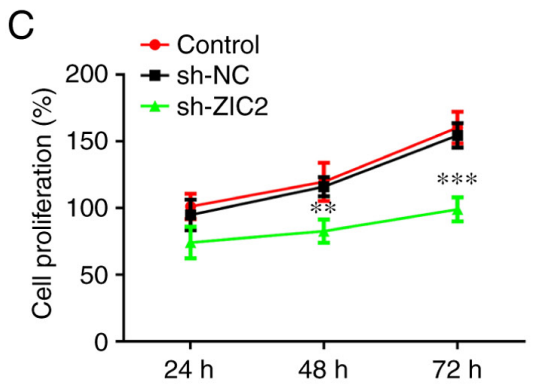
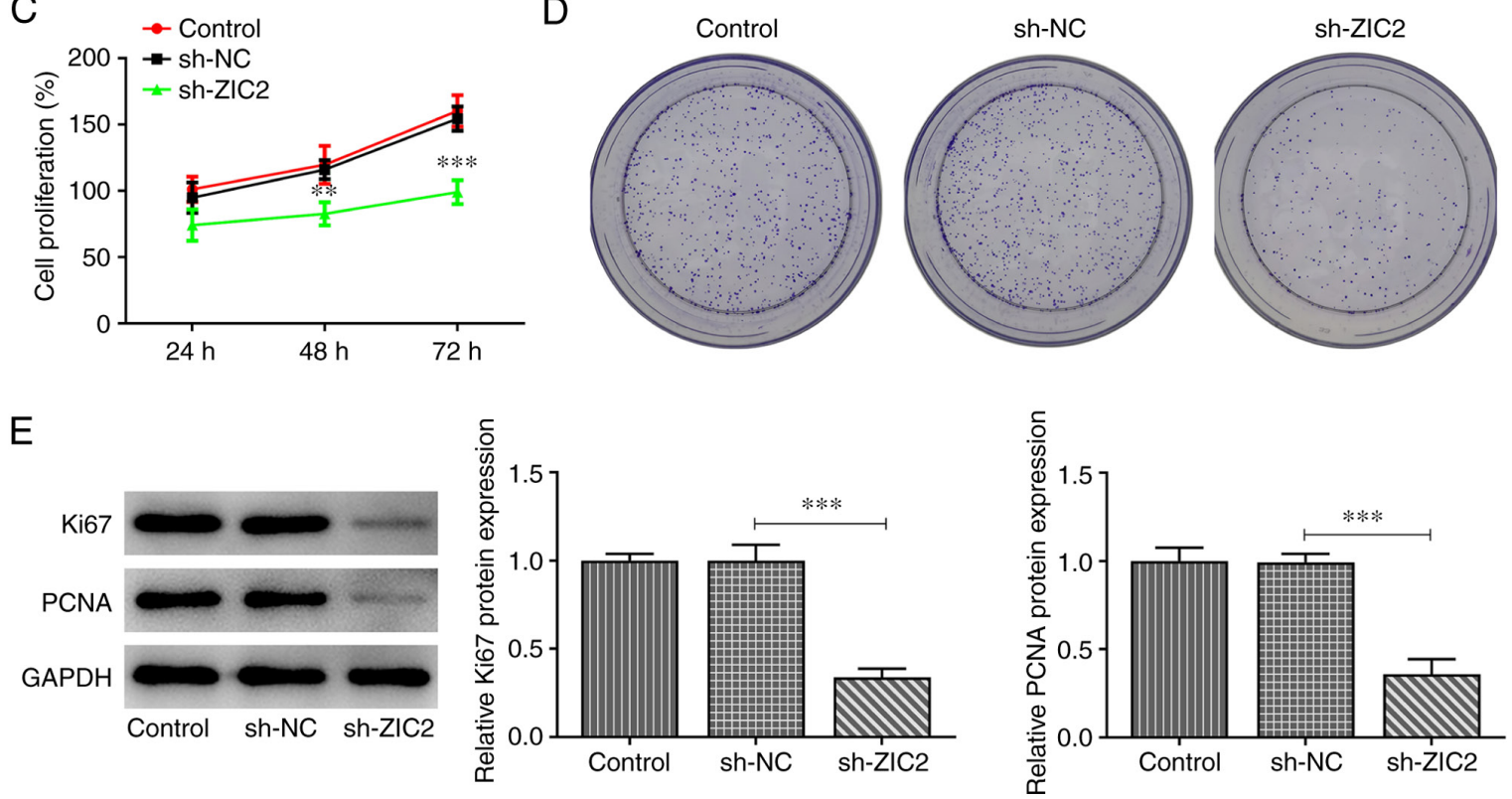

Figure 2. ZIC2 knockdown inhibits RL95-2 endometrial cancer cell proliferation. (A) Protein and (B) mRNA expression levels of ZIC2 in RL95-2 cells before and after transfection with shRNAs. (C) Cell Counting Kit-8 assay was performed to determine the proliferation of RL95-2 cells before and after transfection with shRNA-ZIC2. (D) Representative images for the colony formation assay of RL95-2 cells before and after transfection with shRNA-ZIC2 (magnification, x100). (E) Protein expression levels of Ki67 and PCNA in RL95-2 cells before and after transfection with shRNA-ZIC2 were determined using western blotting. ${ }^{*} \mathrm{P}<0.05,{ }^{* *} \mathrm{P}<0.01,{ }^{* * *} \mathrm{P}<0.001$. ZIC2, Zic family member 2; shRNA, short hairpin RNA; PCNA, proliferating cell nuclear antigen; NC, negative control.

with $1 \%$ formaldehyde for $10 \mathrm{~min}$ at $37^{\circ} \mathrm{C}$. Subsequently, cells were subjected to a ChIP assay according to the manufacturer's protocol of a High-Sensitivity kit (Abcam; cat. no. ab185913). The following antibodies, Anti-ZIC2 (1:1,000; Abcam; cat. no. ab150404) and anti-IgG (as the NC; 1:10,000; Abcam; cat. no. ab133470), were used at $4^{\circ} \mathrm{C}$ overnight. The primer sequences used for the RT-qPCR analysis of SNHG12 expression levels were: Forward, 5'-TCTGGTGATCGAGGACTT CC-3' and reverse, 5'-ACCTCCTCAGTATCACACACT-3'.

Statistical analysis. Statistical analysis was performed using SPSS 19.0 software (IBM Corp.). All experiments were performed at least three times and data are presented as the mean \pm SD. Statistical differences between groups were determined using a one-way ANOVA followed by a Tukey's post hoc test. $\mathrm{P}<0.05$ was considered to indicate a statistically significant difference.

\section{Results}

ZIC2 expression levels are upregulated in patients with EC and EC cell lines. To determine whether ZIC2 was involved in EC, the overall level of ZIC2 differential expression between healthy individuals and cancer patients was analyzed by searching the ENCORI database (15) from The Cancer Genome Atlas (TCGA) Genomics data (16), and the analysis revealed that the expression levels of ZIC2 were significantly upregulated in patients with EC compared with healthy individuals (Fig. 1A). The protein and mRNA expression levels of ZIC2 in the ESC normal endometrial stromal cell line and EC cell lines, Ishikawa, KLE, RL95-2 and AN3 CA, were analyzed. Similarly, the expression levels of ZIC2 were upregulated in EC cell lines, especially in RL95-2 cells, compared with ESC cells (Fig. 1B and C). Therefore, the RL95-2 cell line was selected for use in subsequent experiments as it expressed the highest level of $\mathrm{ZIC} 2$.

Knockdown of ZIC2 inhibits RL95-2 EC cell proliferation, migration and invasion. The expression of ZIC2 was knocked down in RL95-2 cells using shRNA-ZIC2-1 and shRNA-ZIC2-2. As shRNA-ZIC2-1 exerted a greater inhibitory effect on ZIC2 expression, it was selected for use in subsequent studies (Fig. 2A and B). The knockdown of ZIC2 significantly inhibited RL95-2 cell proliferation, 
A
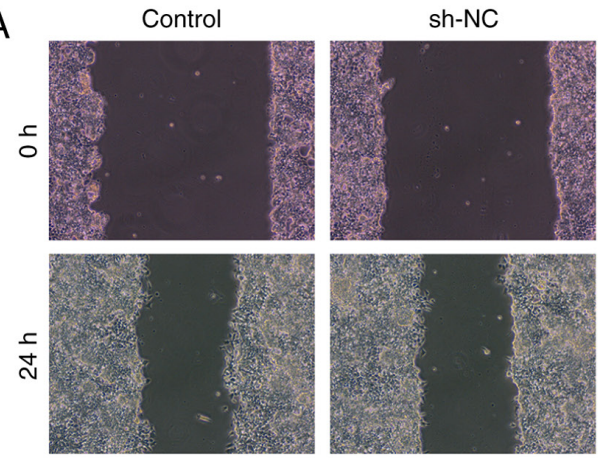

B

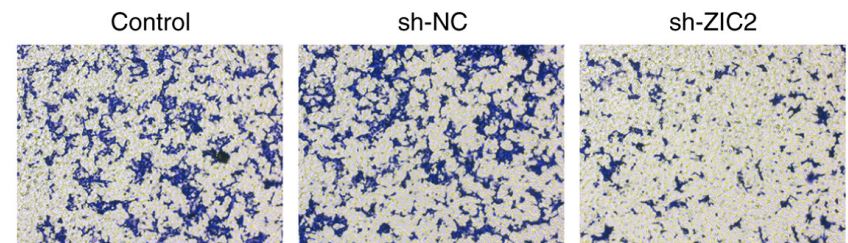

C
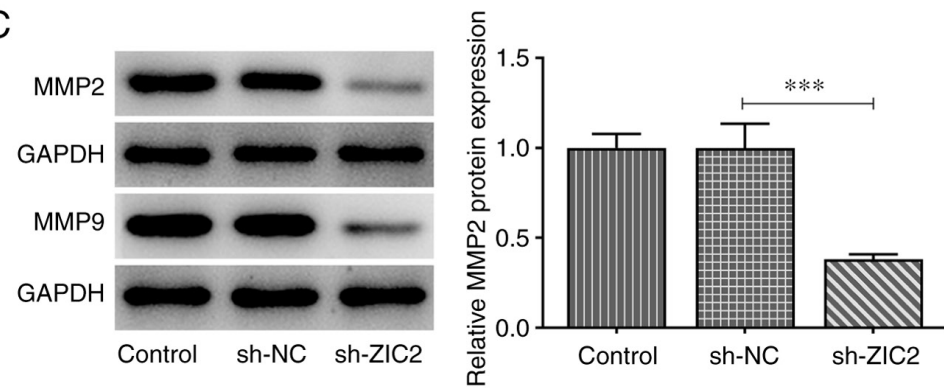

sh-ZIC2

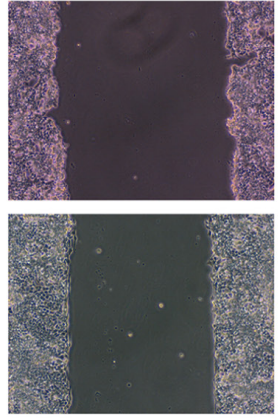

sh-ZIC2
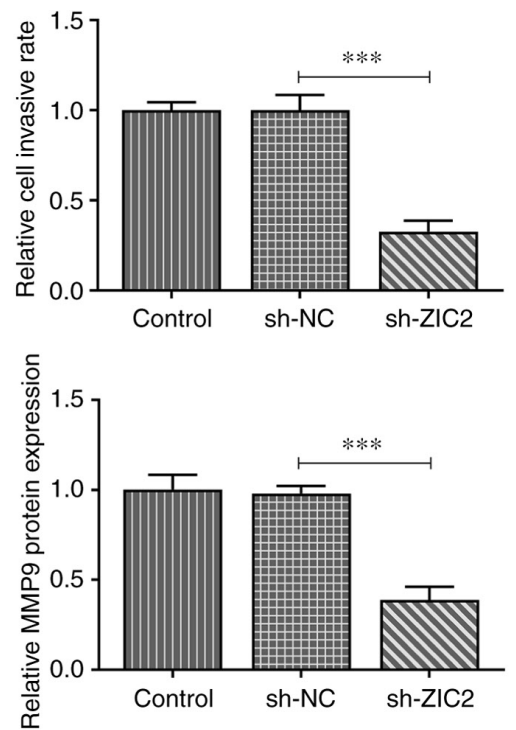

Figure 3. ZIC2 knockdown inhibits RL95-2 endometrial cancer cell migration and invasion. (A) Wound healing and (B) Transwell assays were performed to analyze the migration and invasion, respectively, of RL95-2 cells before and after transfection with shRNA-ZIC2 (magnification, x100). (C) Protein expression levels of MMP2 and MMP9 in RL95-2 cells before and after transfection with shRNA-ZIC2 were analyzed using western blotting. ${ }^{* * *}$ P<0.001. ZIC2, Zic family member 2; shRNA, short hairpin RNA; NC, negative control.

as evidenced by the decreased proliferation rate (Fig. 2C), number of formed colonies (Fig. 2D) and protein expression levels of Ki67 and PCNA (Fig. 2E). The results of the wound healing assay demonstrated that ZIC2 knockdown prevented RL95-2 cells from migrating into the wounded area after $24 \mathrm{~h}$, thus indicating that ZIC2 knockdown was able to inhibit cell migration (Fig. 3A). Transwell assays were used to determine the invasive ability of RL95-2 cells. As demonstrated in Fig. 3B, cells transfected with shRNA-ZIC2 had a significantly lower invasion rate compared with cells in the shRNA-NC and untransfected control groups. In addition, the protein expression levels of MMP2 and MMP9 were downregulated following ZIC2 knockdown (Fig. 3C). These results indicated that ZIC2 knockdown may inhibit RL95-2 cell proliferation, migration and invasion.

ZIC2 binds to SNHG12 and positively regulates its expression. Using the JASPAR database, ZIC2 was predicted to bind to the promoter of SNHG12 (Fig. 4A). Consistent with the results of a recent study (6), SNHG12 expression levels were revealed to be upregulated in EC cell lines, most notably in RL95-2 cells (Fig. 4B). ZIC2 was subsequently overexpressed in RL95-2 cells (Fig. 4C and D) and a dual luciferase reporter assay was performed. The results revealed that ZIC2 overexpression significantly decreased the relative luciferase activity of SNHG12-WT, but not SNHG12-MUT vectors (Fig. 4E), indicating the direct interaction between ZIC2 and SNHG12, which was further validated using a ChIP assay (Fig. 4F). In addition, SNHG12 expression levels in RL95-2 cells were upregulated by ZIC2 overexpression, whereas they were downregulated by ZIC2 knockdown, indicating the positive regulatory effect of ZIC2 on SNHG12 expression (Fig. 4G).

SNHG12 overexpression blocks the effect of ZIC2 on RL95-2 EC cell proliferation, migration and invasion while activating Notch signaling. SNHG12 was overexpressed in RL95-2 cells by transfection with pcDNA 3.1-SNHG12 vectors (Fig. 5A), while ZIC2 expression was also knocked down in RL95-2 cells. Rescue assays were subsequently performed to evaluate whether ZIC2 regulated EC cellular processes by targeting SNHG12. As revealed in Fig. 5B-D, cell proliferation was markedly inhibited following ZIC2 silencing; however, SNHG12 overexpression significantly rescued cell proliferation. Furthermore, ZIC2 knockdown-induced inhibition of RL95-2 cell migration and invasion was partially reversed by SNHG12 overexpression, as evidenced by the increased migration and invasion rates and upregulated expression levels of MMP2 and MMP9 in the cells in the ZIC2 


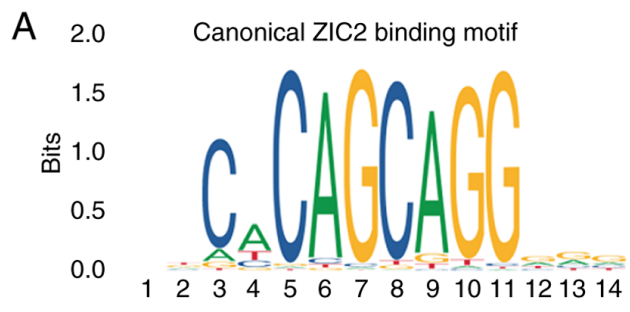

SNHG12 promoter

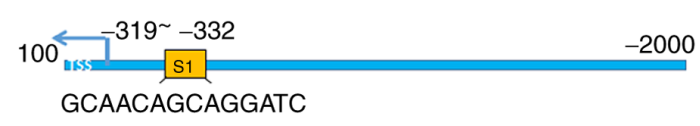

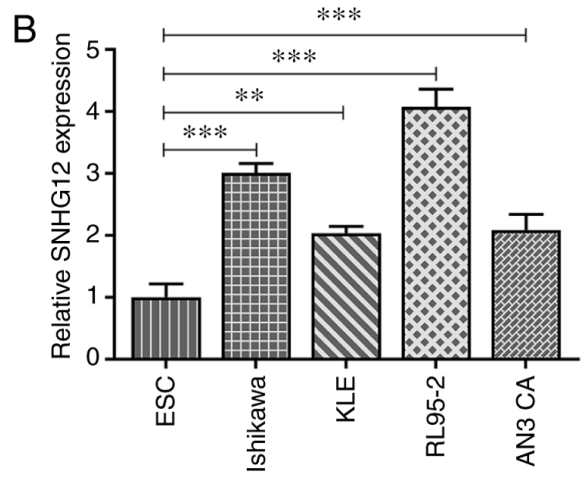

$\mathrm{D}$

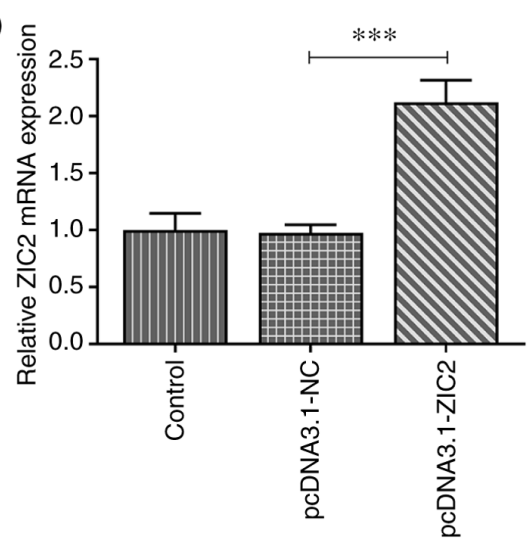

G

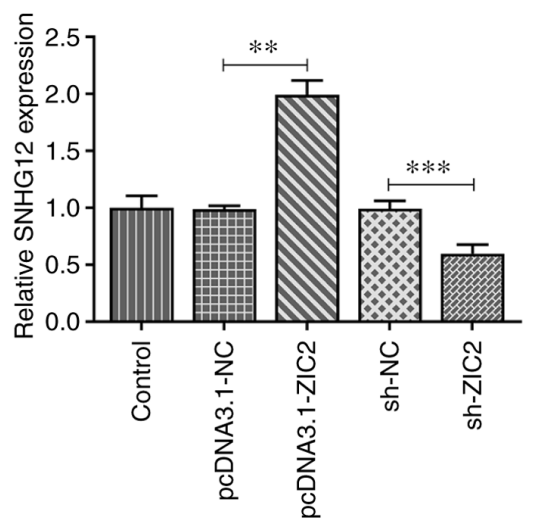

Figure 4. ZIC2 binds to SNHG12 and regulates its expression in RL95-2 cells. (A) Binding sequences between ZIC2 and SNHG12 promoter were predicted using the JASPAR database. (B) mRNA expression levels of SNHG12 in ESC cells and endometrial cancer cell lines. (C) Protein and (D) mRNA expression levels of ZIC2 in RL95-2 cells before and after transfection with pcDNA3.1-ZIC2. (E) Relative luciferase activity of RL95-2 cells co-transfected with the indicated vectors. (F) Relative SNHG12 enrichment in RL95-2 cells subjected to a chromatin immunoprecipitation assay. (G) mRNA expression levels of SNHG12 in RL95-2 cells transfected with the indicated vectors. ${ }^{* *} \mathrm{P}<0.01$ and ${ }^{* * * *} \mathrm{P}<0.001$. ZIC2, Zic family member 2; SNHG12, small nucleolar RNA host gene 12; ESC, endometrial stromal cell; NC, negative control.

knockdown + SNHG12 overexpression group compared with the ZIC2 knockdown + Oe-NC group (Fig. 5E-J).

Finally, the expression levels of proteins associated with Notch signaling were analyzed in RL95-2 cells following ZIC2 knockdown and SNHG12 overexpression to determine whether Notch signaling was involved in the effects of the ZIC2/SNHG12 signaling axis in EC. As revealed in Fig. 5K, the protein expression levels of activated Notch1 and HES-1 were significantly downregulated following ZIC2 knockdown, while they were partially rescued by SNHG12 overexpression, indicating that ZIC2 may activate Notch signaling by upregulating the expression levels of SNHG12 in RL95-2 cells.

\section{Discussion}

Although the 5-year survival rate following early diagnosis and treatment of EC can be as high as $90 \%$, the 5-year survival rate for patients with stage III and IV disease remains low $(4,5)$. Understanding the precise molecular mechanisms of proliferation and metastasis is important for developing improved therapeutic strategies for patients with EC. Therefore, it is pivotal to determine the key molecular mechanisms involved in EC cell proliferation and metastasis. In our previous study, SNHG12 was identified as a biomarker that could be used for the early diagnosis and targeted therapy of EC (6). The 


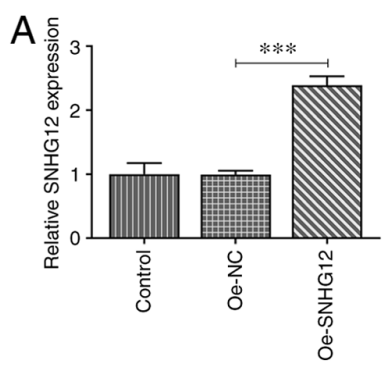

C
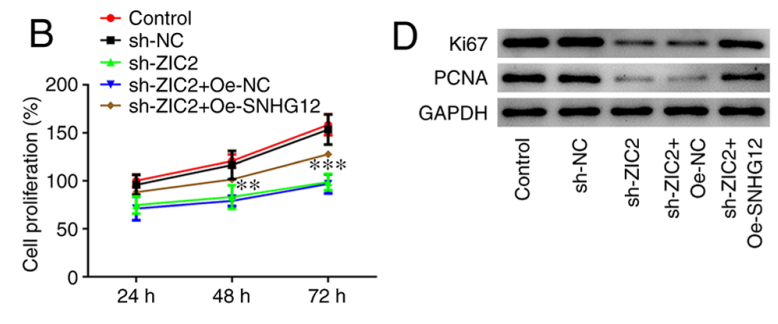

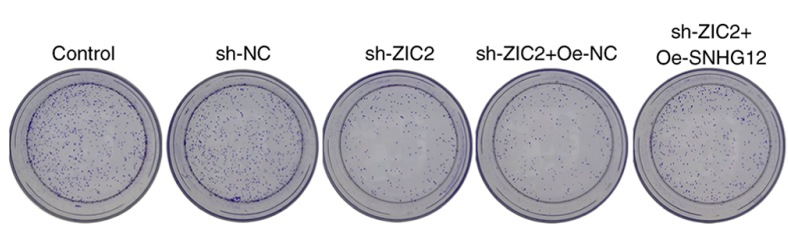

$\mathrm{E}$

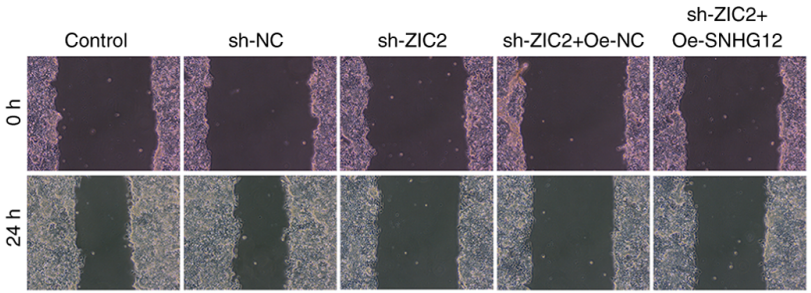

G

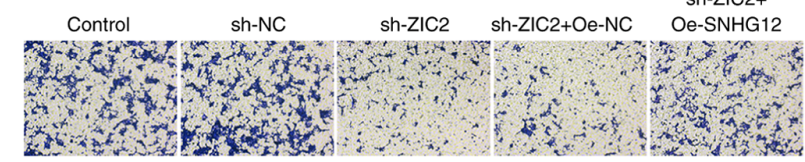

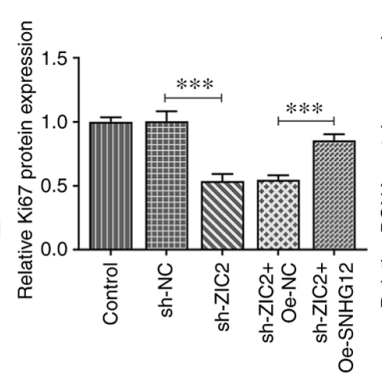

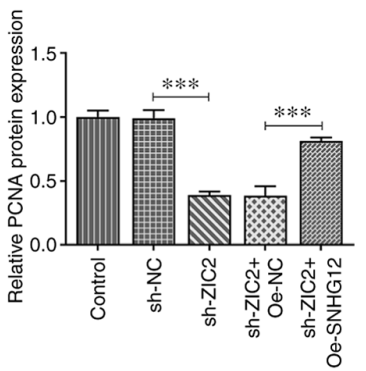

$\mathrm{H}$

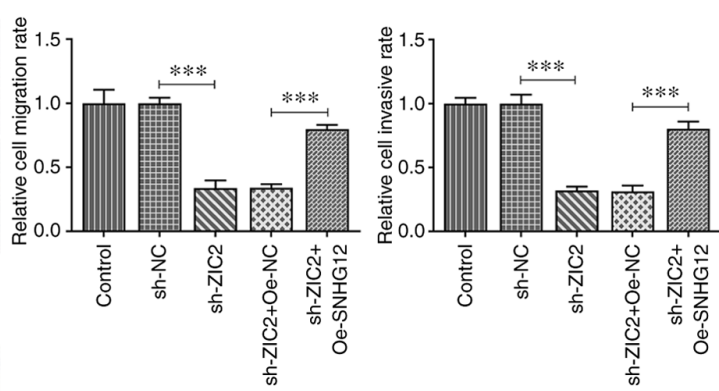

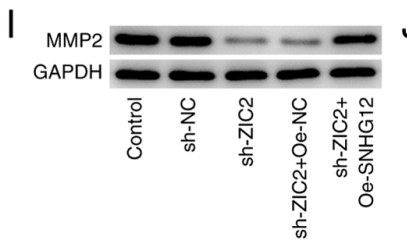

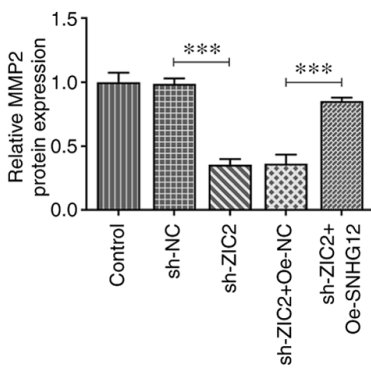

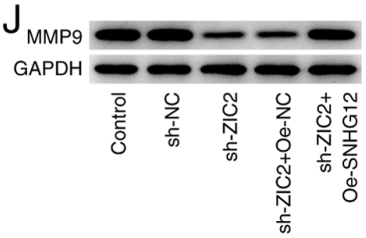

K Activated Notch1
HES-1
GAPDH
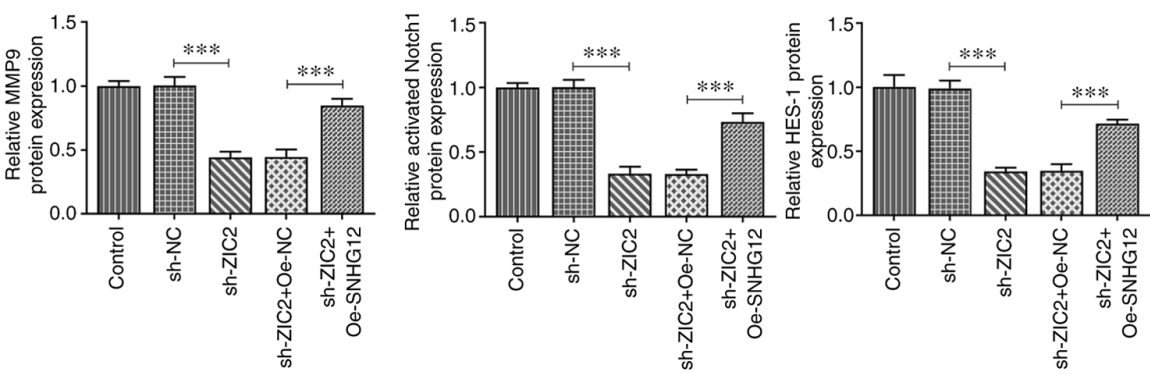

Figure 5. SNHG12 overexpression partially reverses the effect of ZIC2 knockdown in RL95-2 endometrial cancer cells. (A) mRNA expression levels of SNHG12 in RL95-2 cells before and after the overexpression of SNHG12. (B) Cell Counting Kit-8 assay was performed to determine the proliferation of RL95-2 cells before and after transfection with the indicated vectors. (C) Representative images for the colony formation assay of RL95-2 cells before and after transfection with the indicated vectors (magnification, x100). (D) Protein expression levels of Ki67 and PCNA in RL95-2 cells. (E and F) Wound healing and $(\mathrm{G}$ and $\mathrm{H})$ Transwell assays were used to determine the migration and invasion, respectively, of RL95-2 cells before and after transfection with the indicated vectors (magnification, x100). Expression levels of (I) MMP2, (J) MMP9 and (K) Notch signaling pathway-related proteins in RL95-2 cells before and after transfection with the indicated vectors were analyzed using western blotting. ${ }^{* *} \mathrm{P}<0.01$ and ${ }^{* * *} \mathrm{P}<0.001$. SNHG12, small nucleolar RNA host gene 12 ; ZIC2, Zic family member 2; PCNA, proliferating cell nuclear antigen; Oe, overexpression; NC, negative control; sh, short hairpin.

results of the present study demonstrated that the expression levels of ZIC2 were upregulated in patients with EC and EC cell lines, and ZIC2 bound to SNHG12 to positively regulate its expression. Further results revealed that the knockdown of ZIC2 inhibited EC cell proliferation, migration and invasion by regulating SNHG12 expression. Consequently, the ZIC2/SNHG12 signaling axis may serve as a target for novel therapeutic strategies for EC.

The ZIC protein was discovered to be essential for developing vertebrate embryos and numerous human cancer 
types (19). ZIC2 was only found in normal brain and testicular tissues, but was highly expressed in tumors (19). The expression levels of ZIC2 were also revealed to be upregulated and play key roles in multiple types of cancer. For example, ZIC2 knockdown inhibited the proliferation, migration and invasion of breast cancer cells (20). In another previous study, ZIC2 expression was gradually upregulated from normal to cancer to metastatic tissues, and promoted tumor growth and metastasis in HCC (8). ZIC expression was also found to be upregulated in NPC, and knocking down ZIC2 expression was indicated to inhibit NPC lymphangiogenesis and lymph node metastasis (21). The findings of the present study revealed that the expression levels of ZIC2 were upregulated in patients with EC and EC cell lines. In addition, the upregulated expression of ZIC2 is associated with a poor overall survival rate, indicating that ZIC2 may play an oncogenic role in EC (22). ZIC2 expression was subsequently knocked down in EC cells and the results demonstrated that the proliferation rate, colony formation ability, protein expression levels of Ki67, PCNA, MMP2 and MMP9, and the migration and invasion rates of EC cells, were all significantly suppressed following ZIC2 knockdown. According to previous studies, the protein expression of MMP2 and MMP9 along with wound healing and Transwell assays were revealed to be sufficient to indicate the migration and invasion of tumor cells $(23,24)$. Consequently, these results indicated that the knockdown of ZIC2 may inhibit EC cell proliferation, migration and invasion.

ZIC2 was predicted to bind to the promoter of SNHG12, which was demonstrated to promote EC progression in our previous study (6). ZIC2 has been reported to be dominantly located in the nucleus (25). In the present study, dual luciferase reporter and ChIP assays were performed to validate their interaction. ZIC2 was observed to positively regulate SNHG12 expression. It was therefore hypothesized that ZIC2 may bind to SNHG12 to upregulate SNHG12 expression, thereby exerting its stimulatory effect on EC cell proliferation, migration and invasion. SNHG12 was subsequently overexpressed in EC cells with knocked down ZIC2 expression to determine whether the effect of ZIC2 knockdown on EC cells could be modified. Consistent with the present study hypothesis, the effects of ZIC2 knockdown on EC cells were notably reversed by the overexpression of SNHG12. These data indicated that ZIC2 may exert its effects on EC via targeting SNHG12. However, SNHG12 overexpression did not completely reverse the effects of ZIC2 knockdown, suggesting that the oncogenic effects of ZIC2 in EC may also be mediated via other downstream mechanisms.

The present results also revealed that the knockdown of ZIC2 inhibited the activation of Notch signaling, whereas SNHG12 overexpression reversed this effect. SNHG12 has been revealed to modulate the Notch signaling pathway in cancer cells $(26,27)$. In addition, inhibition of the Notch signaling pathway was reported to inhibit the progression of EC $(13,14)$. The present findings identified Notch signaling as the downstream pathway via which SNHG12 regulated EC cell proliferation, migration and invasion. However, future experiments using human samples and animal models need to be performed to validate our findings. The correlation between expression levels of ZIC2 and SNHG12 in EC using clinical human samples will also be analyzed.

In conclusion, the findings of the present study indicated that ZIC2 may act as an upstream mediator of SNHG12 in
EC cells. The knockdown of ZIC2 inhibited EC cell proliferation, migration and invasion via downregulating SNHG12 expression to inhibit Notch signaling. These findings may contribute to the further understanding of the role and mechanism of SNHG12 in EC and indicated that components of the ZIC2/SNHG12 signaling axis may serve as potential diagnostic and prognostic biomarkers, or targets for novel therapeutic strategies for EC.

\section{Acknowledgements}

Not applicable.

\section{Funding}

No funding was received.

\section{Availability of data and materials}

The datasets used and/or analyzed during the present study are available from the corresponding author on reasonable request.

\section{Authors' contributions}

PC and GL acquired the data. MW, HB and BZ contributed to the study design and analysis of the data. MW drafted the manuscript. HB and BZ revised it critically for important intellectual content. HB, PC and GL are responsible for confirming the authenticity if the raw data. All authors read and approved the final manuscript.

\section{Ethics approval and consent to participate}

Not applicable.

\section{Patient consent for publication}

Not applicable.

\section{Competing interests}

The authors declare that they have no competing interests.

\section{References}

1. Sorosky JI: Endometrial cancer. Obstet Gynecol 120: 383-397, 2012.

2. Brooks RA, Fleming GF, Lastra RR, Lee NK, Moroney JW, Son $\mathrm{CH}$, Tatebe $\mathrm{K}$ and Veneris JL: Current recommendations and recent progress in endometrial cancer. CA Cancer J Clin 69: 258-279, 2019.

3. Inoue F, Sone K, Toyohara Y, Takahashi Y, Kukita A, Hara A, Taguchi A, Tanikawa M, Tsuruga T and Osuga Y: Targeting epigenetic regulators for endometrial cancer therapy: Its molecular biology and potential clinical applications. Int J Mol Sci 22: $2305,2021$.

4. Morice P, Leary A, Creutzberg C, Abu-Rustum N and Darai E: Endometrial cancer. Lancet 387: 1094-1108, 2016.

5. Ueda SM, Kapp DS, Cheung MK, Shin JY, Osann K, Husain A Teng NN, Berek JS and Chan JK: Trends in demographic and clinical characteristics in women diagnosed with corpus cancer and their potential impact on the increasing number of deaths. Am J Obstet Gynecol 198: 218.e1-6, 2008. 
6. Cai P, Wu M, Zhang B, Wu S, Wei H and Wei L: Long non-coding RNA SNHG12 regulates cell proliferation, invasion and migration in endometrial cancer by targeting miR-4429. Mol Med Rep 22: 2842-2850, 2020.

7. Bidus MA, Risinger JI, Chandramouli GV, Dainty LA, Litzi TJ, Berchuck A, Barrett JC and Maxwell GL: Prediction of lymph node metastasis in patients with endometrioid endometrial cancer using expression microarray. Clin Cancer Res 12: 83-88, 2006.

8. Lu SX, Zhang CZ, Luo RZ, Wang CH, Liu LL, Fu J, Zhang L, Wang H, Xie D and Yun JP: Zic2 promotes tumor growth and metastasis via PAK4 in hepatocellular carcinoma. Cancer Lett 402: 71-80, 2017.

9. Wang YF, Yang HY, Shi XQ and Wang Y: Upregulation of microRNA-129-5p inhibits cell invasion, migration and tumor angiogenesis by inhibiting ZIC2 via downregulation of the Hedgehog signaling pathway in cervical cancer. Cancer Biol Ther 19: 1162-1173, 2018.

10. Chen X, Yang S, Zeng J and Chen M: miR-1271-5p inhibits cell proliferation and induces apoptosis in acute myeloid leukemia by targeting ZIC2. Mol Med Rep 19: 508-514, 2019.

11. Shen ZH, Zhao KM and Du T: HOXA10 promotes nasopharyngeal carcinoma cell proliferation and invasion via inducing the expression of ZIC2. Eur Rev Med Pharmacol Sci 21: 945-952, 2017.

12. Liu ZB, Tang C, Jin X, Liu SH and Pi W: Increased expression of lncRNA SNHG12 predicts a poor prognosis of nasopharyngeal carcinoma and regulates cell proliferation and metastasis by modulating Notch signal pathway. Cancer Biomark 23: 603-613, 2018.

13. Jonusiene $\mathrm{V}$ and Sasnauskiene A: Notch and endometrial cancer. Adv Exp Med Biol 1287: 47-57, 2021.

14. Shang C, Lang B and Meng LR: Blocking NOTCH pathway can enhance the effect of EGFR inhibitor through targeting CD133+ endometrial cancer cells. Cancer Biol Ther 19: 113-119, 2018

15. Li JH, Liu S, Zhou H, Qu LH and Yang JH: starBase v2.0: Decoding miRNA-ceRNA, miRNA-ncRNA and protein-RNA interaction networks from large-scale CLIP-Seq data. Nucleic Acids Res 42: D92-D97, 2014

16. Chandrashekar DS, Bashel B, Balasubramanya SAH, Creighton CJ, Ponce-Rodriguez I, Chakravarthi BVSK and Varambally S: UALCAN: A portal for facilitating tumor subgroup gene expression and survival analyses. Neoplasia 19: 649-658, 2017.

17. Livak KJ and Schmittgen TD: Analysis of relative gene expression data using real-time quantitative PCR and the 2(-Delta Delta C(T)) method. Methods 25: 402-408, 2001.
18. Chen C, Shao R, Li B, Zhai Y, Wang T, Li X, Miao L, Huang J, Liu R, Liu E, et al: Neoisoliquiritin exerts tumor suppressive effects on prostate cancer by repressing androgen receptor activity. Phytomedicine 85: 153514, 2021

19. Zhu P, Wang Y, He L, Huang G, Du Y, Zhang G, Yan X, Xia P, Ye B, Wang S, et al: ZIC2-dependent OCT4 activation drives self-renewal of human liver cancer stem cells. J Clin Invest 125: 3795-3808, 2015.

20. Zhang P, Yang F, Luo Q, Yan D and Sun S: miR-1284 inhibits the growth and invasion of breast cancer cells by targeting ZIC2 Oncol Res 27: 253-260, 2019.

21. Yu D, Han GH, Zhao X, Liu X, Xue K, Wang D and Xu CB: MicroRNA-129-5p suppresses nasopharyngeal carcinoma lymphangiogenesis and lymph node metastasis by targeting ZIC2. Cell Oncol (Dordr) 43: 249-261, 2020.

22. Lv Z, Qi L, Hu X, Mo M, Jiang H, Fan B and Li Y: Zic family member 2 (ZIC2): A potential diagnostic and prognostic biomarker for pan-cancer. Front Mol Biosci 8: 631067, 2021.

23. Xu F, Li Q, Wang Z and Cao X: Sinomenine inhibits proliferation, migration, invasion and promotes apoptosis of prostate cancer cells by regulation of miR-23a. Biomed Pharmacother 112: 108592, 2019

24. Wan G, Liu Y, Zhu J, Guo L, Li C, Yang Y, Gu X, Deng LL and $\mathrm{Lu}$ C: SLFN5 suppresses cancer cell migration and invasion by inhibiting MT1-MMP expression via AKT/GSK-3 $\beta / \beta$-catenin pathway. Cell Signal 59: 1-12, 2019.

25. Marchini S, Poynor E, Barakat RR, Clivio L, Cinquini M, Fruscio R, Porcu L, Bussani C, D'Incalci M, Erba E, et al: The zinc finger gene ZIC2 has features of an oncogene and its overexpression correlates strongly with the clinical course of epithelial ovarian cancer. Clin Cancer Res 18: 4313-4324, 2012.

26. Zhou S, Yu L, Xiong M and Dai G: LncRNA SNHG12 promotes tumorigenesis and metastasis in osteosarcoma by upregulating Notch2 by sponging miR-195-5p. Biochem Biophys Res Commun 495: 1822-1832, 2018.

27. Zhang L, Wan Y, Zhang Z, Jiang Y, Lang J, Cheng W and Zhu L: FTO demethylates m6A modifications in HOXB13 mRNA and promotes endometrial cancer metastasis by activating the WNT signalling pathway. RNA Biol: Nov 5, 2020 (Epub ahead of print).

This work is licensed under a Creative Commons Attribution-NonCommercial-NoDerivatives 4.0 International (CC BY-NC-ND 4.0) License. 